\title{
Helioseismic observations of magnetic flux emergence and flare effects
}

\author{
A. G. Kosovichev ${ }^{1}$ and T. L. Duvall, $\mathrm{Jr}^{2}$ \\ ${ }^{1}$ Stanford University, Stanford, CA 94305, USA; ${ }^{2}$ NASA GSFC, Greenbelt, MD 20771, USA
}

\begin{abstract}
Time-distance helioseismology and data from SOHO/MDI are used for obtaining $3 \mathrm{D}$ images of subsurface sound-speed perturbations and maps of plasma flows, associated with emerging magnetic flux and flaring activity of large active regions in October 2003. The results reveal extremely complicated dynamical processes in the upper convection zone and indicate that subsurface shear flows may play an important role in magnetic energy release in solar flares. Strong X-class flares generated impulsive seismic waves ("sunquakes"), traveling through surrounding sunspots, thus providing new insight into the interaction of seismic waves with magnetic fields.
\end{abstract}

Keywords. Sun: helioseismology, interior, magnetic fields, oscillations, sunspots, flares, particle emission, X-rays, gamma rays

\section{Introduction}

New intensively developed methods of local helioseismology provide unique information about the structure and dynamics of active regions below the Sun's surface (e.g. Kosovichev et al., 2000; Haber et al., 2004; Zhao et al., 2001). These methods provide 3D images of sound-speed perturbations and maps of flow patterns beneath developing active regions, and allow us to investigate the relationship between the sub-surface dynamics and evolution and structure of the photospheric magnetic fields. The initial results suggest a strong coupling between the sub-surface flows and processes of magnetic energy release in the solar atmosphere. Here, we present some results obtained by time-distance helioseismology (Duvall et al., 1993), using SOHO/MDI data (Scherrer et al., 1995) for a large complex of activity observed in October-November 2003. Uninterrupted observations from SOHO during this period allowed us to obtain subsurface images of emerging active region, NOAA 10488, observe subphotospheric flow patterns in AR 10486 during the period of large flares and compare these with the magnetic field changes during these flares, and finally, detect traveling helioseismic waves excited by these flares.

\section{Observations of an Emerging Active Region in the Sun's Interior}

Active region NOAA 10488 emerged on October 26, 2003, about 12 UT. It appeared in the Northern hemisphere approximately $30^{\circ}$ North of AR 10486, which was in the Southern hemisphere. Figure 1 shows depth cuts through the images of the sound-speed variations associated with this active region, shortly after the emergence, (Fig. 1a) and three days later when the active region is fully developed (Fig. 1b). These images show the typical structure: reduced sound speed regions, 4-5 Mm deep, below the sunspots, and higher sound-speed regions in the deeper interior. In Fig. 1a, showing a vertical cut through both active regions (approximately in the North-South direction) there is evidence for a large deep structure between the old active region, NOAA 10486, and the new one, 10488. This could be an indication of a possible deep connection in this 

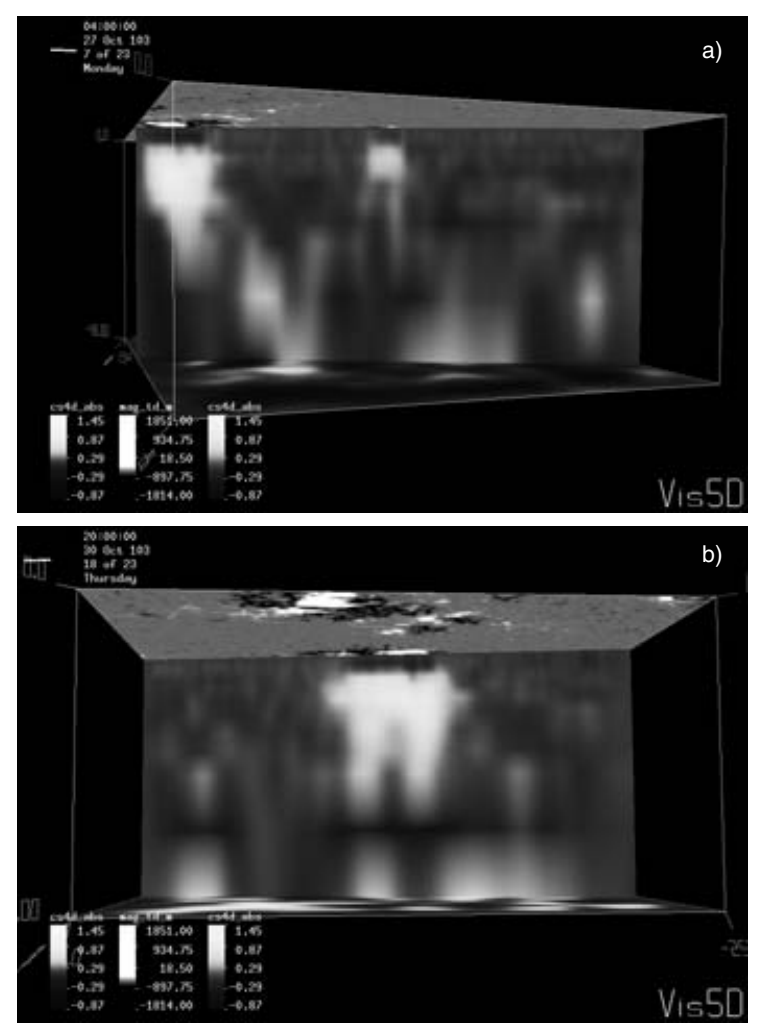

Figure 1. Sound-speed variations (vertical cuts) associated with developing active region NOAA 10488 (October 2003): a) emergence of the active region in the middle of the domain (the structure near the right boundary is AR10486; b) fully developed AR 10488. The depth of the box is $48 \mathrm{Mm}$, the horizontal size is about $540 \mathrm{Mm}$. The sound-speed scale is from -1 to $1.5 \mathrm{~km} / \mathrm{s}$ (shown in gray from dark to light), the scale of the photospheric magnetic field shown in the upper panel (view from below the surface) is from -1800 to 1800 Gauss.

complex of activity. However, the roots of active regions have not been detected. They are probably deeper than the current probing depth of this method. Figure 1b, a East-West cut through AR10488, reveals a loop-like structure, which seem to extend into the layers below the lower boundary.

\section{Sub-photospheric Shear Flows and Energy Release in Solar Flares}

A series of 9 X-class flares produced during Oct.23-Nov.4, 2003 by the two active region 10486 and 10488 was one of the most powerful in the history of solar observations. It is well-known that flares usually occur in complex sheared and twisted magnetic configurations which are presumably produced by shearing and twisting plasma flows below the surface where the dynamic pressure of plasma flows may exceed the magnetic pressure. Magnetic energy release in solar flares typically happens around neutral lines of the lineof-sight (vertical) component of magnetic field. These places can be identified by rapid permanent changes of the photospheric magnetic flux on both sides of the neutral line (Kosovichev \& Zharkova, 2001). Presumably the energy is released mostly in magnetic structures in the upper atmosphere, covering some range of heights, but evidently these structures are connected to the places near the magnetic neutral line in the photosphere 

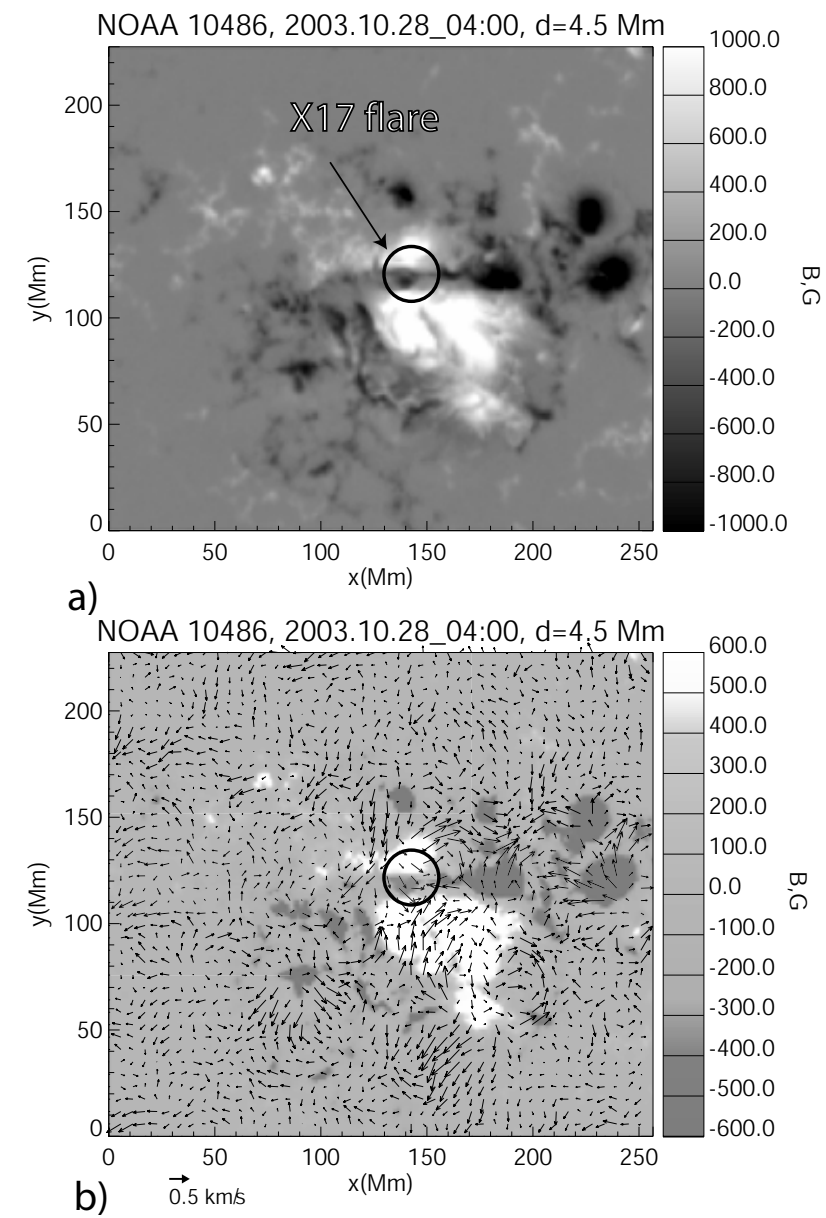

Figure 2. Surface magnetograms and subsurface flows during the X17 flare of Oct. 28, 2003.

where we see significant permanent changes of the line-of-site component of magnetic field during the impulsive phase of solar flares.

The black circles in Figure 2 indicate the site of the magnetic energy release of a strong flare, X17 started at 9:51 UT on October 28 in AR10486, where a significant permanent change of the field strength was detected during the impulsive phase. It is intriguing that the flow maps inferred by time-distance helioseismology at the depth of 4-6 Mm reveal strong (with speed about 1-2 km) shearing flows directed to the sites of the magnetic energy release during these flares. This is particularly evident from the flow map (Fig. 2b, taken for the 8-hour periods: 0-8 UT on October 28 (labeled as 2003.10.28_04:00, just before the X17 flare.

\section{Helioseismic Effects of Solar Flares}

It is interesting that the same X17 flare generated impulsive circular waves, "sunquakes" (Fig. 3) (Kosovichev, 2005). These waves were excited by the hydrodynamic impact of atmospheric plasma heated to high temperatures by energetic electrons accelerated in the upper atmosphere during the magnetic energy release. The centers of the hydrodynamic impact are identified during the flare impulsive phase as strong localized 

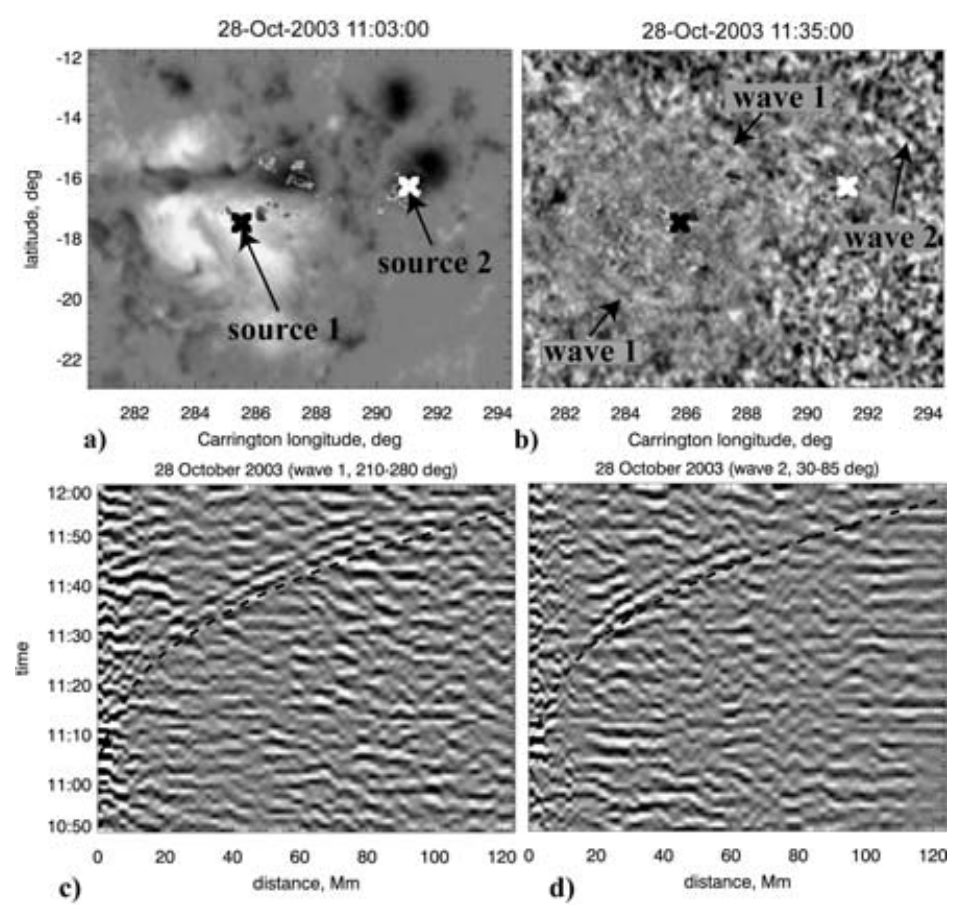

Figure 3. Observations of the seismic response to X17 flare of October 28, 2003: a) the smooth background image is the SOHO/MDI magnetogram taken at the beginning of the flare, 11:03 UT; small localized patches on this image are Doppler velocity variations greater than $1 \mathrm{~km} / \mathrm{s}$; black and white crosses show the central locations of two strongest seismic waves excited by this flare; b) the MDI Dopplergram temporal first difference at 11:35 UT, showing partial circular wavefronts; c) and d) the time-distance diagrams for the two waves, obtained by averaging the Doppler velocity signal in the azimuthal angle range, 210-280 deg (counting from West), for wave 1, traveling South-East, and in the range of 30-85 deg., for wave 2, traveling North-West; the dashed curves are theoretical time-distance relations for solar acoustic waves in the ray approximation.

rapidly varying velocity perturbations of about $1 \mathrm{~km} / \mathrm{s}$ (small gray patches in Fig. 3a overlying the MDI magnetogram). The crosses in Fig.1a and b show the precise location of the centers of the sunquakes, which are close to the sources of hard X-ray emission, but far from the neutral line, where the flare originated (Fig. 2). The time-distance relation of these acoustic waves (Fig. 1c and d) is very close to the theoretical time-distance relations (dashed curves) for the standard solar model. This means that regions of strong magnetic field, such as sunspots, only weakly affect the travel times of the acoustic waves. Also, we do not observe a significant change in the amplitude of these as they traveled through the magnetic regions, suggesting that there was no significant absorption or conversion of these waves into other types of MHD waves.

\section{References}

Duvall, T. L., Jr., S. M. Jefferies, J. W. Harvey \& M. A. Pomerantz 1993, Nature 362, 430.

Haber, D.A., B. W. Hindman, J. Toomre, \& M. J. Thompson, 2002, Solar Phys. 220, 371.

Kosovichev, A. G., T. L. Duvall, Jr. and P. H. Scherrer 2000, Solar Phys. 192, 159.

Kosovichev, A.G. \& V.V. Zharkova, 2001, Astrophys.J. 550, L105.

Kosovichev, A. G., 2005, astro-ph/0601006.

Scherrer, P. H., et al. 1995, Solar Phys. 162, 129.

Zhao, J., A. G. Kosovichev and T. L. Duvall, Jr. 2001, Astrophys. J. 557, 384. 